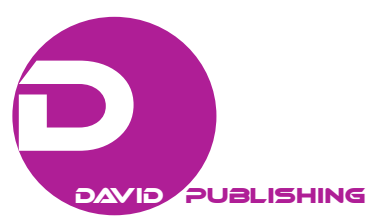

\title{
A Corpus-based Contrastive Study on the Acquisition of Synonyms of Chinese EFL Learners
}

\author{
LI Xiang, LIU Juan \\ Guangdong Police College, Guangzhou, China
}

\begin{abstract}
This paper investigates Chinese EFL learners' acquisition of completely, totally and absolutely through a contrastive analysis of their collocation, colligation, semantic preference and semantic prosody based on the Ten-thousand English Compositions of Chinese Learners (TECCL Corpus) and Corpus of Contemporary American English (COCA). The results show that adjective and verb are two major words frequently co-occurring with the group of synonyms, but only a few of them meet the default collocation criterion (frequency $\geq 3, \mathrm{MI} \geq 3$ ), and many co-occurring words used by Chinese learners don't appear in COCA. Besides, the co-occurring adjectives and verbs in TECCL are so diverse that they don't establish fixed semantic relations as in COCA; therefore, there is a big difference in semantic preference and semantic prosody between Chinese learners and native speakers. With the advance of English proficiency, more collocates are used by college learners than middle school learners, but there is no clear and significant improvement in semantic preference and semantic prosody.
\end{abstract}

Keywords: synonyms, corpus, contrastive study, semantic preference, semantic prosody

\section{Introduction}

Synonyms refer to the words or expressions with the same meaning. The use of synonyms is not only an important way to enrich texts, but helps EFL learners to construct vocabulary net and build semantic relations with acquired words, so they have always been the focus of EFL teaching and learning and studying. However, the previous studies have shown that although synonyms or near synonyms have very similar cognitive or denotational meanings, they may differ in collocational or prosodic behavior, that is to say, synonymous words are not collocationally interchangeable and they may demonstrate not only different collocational behaviour but also distinct semantic prosodies (Partington, 1998; XIAO \& McEnery, 2006; Gardiner \& Dras, 2007). These subtle differences between synonyms, which are usually distinguished by native speakers' language sense and intuition, are not clearly and completely explicated in the dictionary, so they particularly pose a difficulty for most EFL learners. In recent years some scholars in China have made significant researches on the differences of synonyms based on English corpora such as COCA and BNC (ZHANG \& LIU, 2005; PANG \& YANG, 2012; FANG, 2012), but only a few attentions have been paid to the synonyms used by Chinese English learners, let 
alone the development of synonym acquisition. This present study is to investigate how Chinese English learners use and discriminate synonyms based on interlanguage corpora of different learning stages.

\section{Literature Review}

Since Firth (1957, p. 11) famously stated "you shall know a word by the company it keeps", Post-Firthian linguistics' researches on meaning have shifted from single word to lexical item (Sinclair, 1996), lexical chunks (Lewis, 1993), patterns (Hunston \& Thompson, 2000) or phrase (Sinclair, 2008) with the development of corpus linguistics. No matter what terms are invented, almost all corpus linguistists emphasize the combination of lexis and grammar and meaning by contextual relations. In order to search the units of meaning, Sinclair (1996) set a model which includes five categories of co-selection, that is, core, collocation, colligation, semantic preference and semantic prosody, for short, "3C2S". In this model, the obligatory categories are the core, which is invariable, and constitutes the evidence of the occurrence of the item as a whole, and the semantic prosody, which is the determiner of the meaning of the whole (Sinclair, 1996). Collocation refers to the relationship between lexical item and other lexical items. But in corpus linguistics collocation only refers to statistically significant co-occurrence of two lexical items. Hoey (1991, pp. 6-7) uses the term collocation only if a lexical item appears with other items: "with greater than random probability in its (textual) context". Colligation refers to the relationship between lexical item and a grammatical category; semantic preference refers to the semantic categories shared by the frequent collocates of a specific node item (Hunston, 2002; Partington, 2004); Louw (1993, p. 157) firstly defined semantic prosody as a form of meaning which is established through the proximity of a consistent series of collocates, often able to be characterized as positive or negative, and whose primary function is to express the feelings of its speaker or writer towards some pragmatic situation. Hunston and Thompson (2000, p. 5) regarded semantic prosody as "the speaker or writer's attitude or stance towards, viewpoint or feelings about the entities and propositions that he or she is talking about". Sinclair (2003) also noted that semantic prosody conveys its pragmatic meaning and attitudinal meaning. Stubbs (1996, p. 76) divided semantic prosody into three type: negative prosody, neutral prosody and positive prosody. No matter how semantic prosody is defined, it is no doubt that semantic prosody results from the frequent co-occurrence of the core or the node and its collocates.

Considering the model's deep exposure of the distributional characteristics of lexical item, many researchers have applied it especially in the domain of synonymy. Researches have found that each lexical item has its unique colloational behaviors, without the exception of synonyms (Partington, 1998, p. 27; Conzett, 1997, pp. 70-87). Besides, more and more researchers have proven that synonyms have different semantic preference and semantic prosody (Tognini-Bonelli, 2001; Kayaoğlu, 2013; HU, 2015).

In recent years, many studies on synonyms in China have also adopted the model to investigate the differences of synonymous words. These researches are majorly divided into two types: one focuses on the usage of synonyms used by native speakers based on native language corpora with an aim to explore the different co-selection relations of synonyms; the other makes contrastive study on synonyms through the comparison of Chinese learners's interlanguage and native language. WEI (2006) investigated the words commit, cause and effect, based on learner corpus and native speaker's corpus, and found that Chinese EFL learners had a narrow range of collocations, vague semantic meanings, underused or overused semantic prosody. PAN (2010) made a 
contrastive analysis of the collocational features of "cause" and "lead to" in SWECCL (Spoken and Written English Corpus of Chinese Learners) and BNC (British National Corpus) and found that English-major learners demonstrated similar semantic preferences with the native speakers, but that there were still great differences in their underlying collocational patterns. LU (2010) explores the collocational behavior and semantic prosody of near synonyms through a corpus-based contrastive analysis between Chinese learners' English (CLE) and native English and found that CLE exhibits much deviation in both dimensions and different types of CLE exhibit varying degrees of synonymous substitution and prosodic clash.

A research on the published papers finds that studies on synonyms in Chinese Learners' English pay more attention to cross-sectional synonymous behaviors rather than longitudinal development of synonym acquisition. The present study not only aims to explore the difference of synonyms' collocation behavior and semantic prosody between Chinese EFL learners and native speakers, but also investigate the development of synonym acquisition.

\section{Method and Research Questions}

Featuring a wide range of topics or prompts, the Ten-thousand English Compositions of Chinese Learners (TECCL Corpus) was chosen as the Chinese English learner corpus, which contains approximately 10,000 writing samples of Chinese EFL learners, totaling 1,817,472 words from 2010-2015. The writers in the corpus run the gamut from elementary school to postgraduate students, undergraduates being the overwhelming majority; therefore, it can be used to do a longitudinal research on the development of synonym acquisition. The present study chose Middle School sub-corpus and College sub-corpus as the target corpora, for short, the two are labeled as Middle School and College. The sub-corpus of COCA 2010-2015 containing 121.6 million tokens was selected as the reference corpus, which, for short, was labeled as "COCA" in this paper. The data needed is retrieved from the two corpora respectively with the online search tool CQP web in the study.

The group of words "totally, completely, absolutely,", which is called maximizers by Quirk et al (1985), a subset of amplifying intensifiers, is chosen as the target synonyms in this paper and a data-driven approach and Contrastive Interlanguage Analysis were applied in the study. The MI score was used here to measure the collocational strength of node word and its collocates. In this study, within a 4-4 window span, items which have a minimum co-occurrence frequency of three in TECCL and ten in COCA and a minimum MI score of three are considered to be collocates of a node word.

This study explores the use and acquisition of the group of synonyms by Chinese English learners. It tries to answer the following questions:

(1) Do Chinese learners share similar collocational behaviors and semantic prosody with native speakers when using the target group of synonyms? If differences do exist, what are they and what are the reasons?

(2) Is there any acquisition development in using the group of synonyms from middle school learners to college learners? If development exists, what feature it show? 


\section{Results and Discussion}

\section{A general Discription of the Synonyms in Different Corpora}

The comparison among the synonyms is carried out from the perspectives of the frequency of the node, collocation, colligation, semantic preference and semantic prosody. Table 1 shows that the most frequently used words of the group of synonyms in Chinese learner English are different from native speakers. Chinese learners prefer to use completely, absolutely and totally in the order of the frequency ratio, but native speakers prefer to absolutely, completely and entirely. In addition, native speakers use entirely more frequently than Chinese learners. A comparison between middle school learners and college learners exhibits that with the increase of English proficiency level, Chinese learners decrease using completely and increase the use of absolutely instead.

Table 1

The Frequency and Ratio of the Synonyms in Different Corpora (Freq: Instances Per Million Words)

\begin{tabular}{lccccccccc}
\hline Synonym & \multicolumn{3}{c}{ Middle School } & \multicolumn{3}{c}{ College } & \multicolumn{3}{c}{ COCA } \\
\hline & Instances & Freq. & Ratio & Instances & Freq. & Ratio & Instances & Freq. & Ratio \\
Completely & 28 & 65.25 & $47 \%$ & 107 & 69.92 & $33 \%$ & 10331 & 85.11 & $30 \%$ \\
Absolutely & 15 & 37.29 & $25 \%$ & 88 & 63.38 & $32 \%$ & 12142 & 100 & $35 \%$ \\
Totally & 14 & 34.96 & $23 \%$ & 105 & 69.26 & $27 \%$ & 5250 & 43.25 & $15 \%$ \\
\hline
\end{tabular}

\section{Comparison on the Synonyms of Different Corpora}

The investigation on the colligation of the synonyms finds that the major types of colligation of the synonyms used by both Chinese learners and native speakers are as follows: "be + synonym + adj."; "Verb + synonym / synonym + Verb", "(article) + synonym + adj. + Noun". Therefore, Verbs and adjectives are two frequently co-occurring words and will be explored in the following part of the paper.

\section{High frequency adjectives collocating with the synonyms.}

\section{Completely}

Table 2

Adjectives Co-occurring With Completely

\begin{tabular}{lccllllllllc}
\hline \multicolumn{2}{c}{ Middle School } & \multicolumn{3}{c}{ College } & \multicolumn{5}{c}{ COCA } \\
\hline Collocate & Freq & MI & Collocate & Freq & MI & Collocate & Freq & MI & Collocate & Freq & MI \\
Different & 3 & 3.3 & Opposite & 3 & 5.2 & Controllable & 17 & 8.0 & Unaware & 22 & 5.9 \\
New & 2 & 2.2 & Different & 9 & 2.7 & Untrue & 15 & 7.6 & Inappropriate & 31 & 5.8 \\
Great & 1 & 1.5 & New & 7 & 2.7 & Unfounded & 10 & 7.1 & Irrelevant & 17 & 5.8 \\
Real & 1 & 4.1 & Unique & 2 & 4.8 & Devoid & 16 & 6.9 & Ignorant & 10 & 5.6 \\
Serious & 1 & 3.8 & Equal & 2 & 4.5 & Unrealistic & 17 & 6.6 & Useless & 16 & 5.5 \\
Stupid & 1 & 7.4 & Disgusted & 1 & 8.5 & Unrelated & 19 & 6.4 & Ridiculous & 36 & 5.4 \\
Helpless & 1 & 8.9 & Self-reliant & 1 & 7.5 & Unacceptable & 24 & 6.3 & Bald & 14 & 5.3 \\
& & & Understandable & 1 & 7.2 & Irrational & 14 & 6.3 & Unnecessary & 16 & 5.3 \\
& & & Effective & 1 & 3.3 & Oblivious & 12 & 6.1 & Transparent & 17 & 5.2 \\
& & & Big & 2 & 2.0 & Helpless & 16 & 6.0 & Different & 659 & 5.2 \\
\hline
\end{tabular}


The statistic results show that only different in Middle school sub-corpora and opposite in College sub-corpora meet the default standard for collocating frequency $(\geq 3)$ and minimum MI score $(\geq 3)$. So in order to explore the whole collocational profile of completely used by Chinese learners, all the co-occurring words used in interlanguage corpus TECCL are listed in Table 2. As shown in this table, only 7 adjectives are used by middle school learners and 10 adjectives are used by college learners. However, there are 52 statistically significant collocates in COCA. Owing to limited space, only the top 20 high collocability collocates are listed in MI order in Table 2. Besides, the statistics in Table 1 show that the frequency of completely in Middle School sub-corpus, College sub-corpus and reference corpus COCA are 65.25, 69.92 and 85.11 instances per million words respectively, which means native speakers much more frequently use completely than Chinese learners. Therefore, it can be concluded that compared to native speakers, Chinese learners underuse collocates of completely quantitatively and statistically.

Shifting from statistics to concrete collocates, the author finds that, just as the boldfaced words show, 14 collocates have "negative affix" among the top 20 high collocability collocates in COCA. The overwhelming majority of negative affix words demonstrate that when native speakers want to emphasize "negation", they tend to use completely as an amplifying intensifier to express their attitude and emotion. However, this prominent feature does not exist in Chinese learners' interlanguage.

The next step of searching units of meaning is to explore semantic preference, which is defined by Stubbs (1996, p. 65) as "the relation, not between individual words, but between a lemma or word-form and a set of semantically related words". So firstly, it is necessary to investigate how collocates are semantically related, in another word, what kind of meaning is shared by the collocates of the same node. As shown in Table 2, the meanings of the collocates in Chinese learners' language are diverse, that is, the limited collocates hardly form a group of semantically related words. However, the collocates are clearly divided into several semantic groups in COCA. The major semantic group expresses the meaning of "absence" or "lack of", which includes devoid, hopeless, oblivious, bald, naked, unfounded, ignorant, and unaware. The second semantic group including untrue, unrealistic, inappropriate, irrational, unnecessary and unacceptable expresses the meaning of "no practical value". The third group containing unrelated, irrelevant expresses the meaning of "uncorrelation". A comparison of the semantic relation of completely between Chinese learners and native speakers clearly shows that no semantic preference came into existence in Chinese learners' language because no meaning arising from the shared semantic features of the collocates of a given node, which exposes the non-nativeness of the use of completely by Chinese learners. When comparing middle school learners and college learners, the author finds that Chinese learners have not made any significant improvement with the development of English proficiency except the tiny increase of co-occurring words.

Because semantic preference contributes powerfully to building semantic prosody (Partington, 2004, p. 151), the difference in the semantic preference between Chinese learners and native speakers certainly results in different semantic prosodies. As shown in Table 2, 17 out of top 20 high frequency collocates are clearly negative collocates in COCA. They are untrue, unfounded, devoid, unrealistic, unrelated, unacceptable, irrational, oblivious, helpless, unaware, inappropriate, irrelevant, ignorant, useless, ridiculous, bald, unnecessary. The dominating ratio of negative collocates naturally generates a negative prosody in the co-text of completely. However, in Middle School, the unique statistically significant collocate "different" is a neutral collocate. Even if 
taking all the 7 collocates into consideration, 3 positive collocates, new, great, real, take up about 43\%; 2 negative collocates, stupid and helpless, account for around 28.5\%; the rest 2 neutral collocates different and serious take up $28.5 \%$. In College, the majority of the total collocates are positive words such as new, equal, understandable, effective, self-reliant, so college learners convey a positive semantic prosody when using completely.

\section{Absolutely}

Only 9 adjectives co-occur with absolutely within a L4-R4 window span in Middle School, they are different, popular, necessary, fair, expensive, fantastic, nice, poor, right. However, no co-occurring words can be regarded as real collocates because only different appears two times and all the other words appear once. In College, 21 adjectives co-occur with absolutely, but only essential and necessary meet the criterion (frequency $\geq 3, \mathrm{MI} \geq 3$ ). The rest words are deficiency in either collocability or collocating frequency, including different, wrong, harmful, convenient, moral, appalling, infeasible, depressing, affluent, hostile, supportive, prevailing, disastrous, irrational, unnecessary, unforgettable, meaningful and possible. However, there are 39 adjectives meeting the default collocation standard in COCA (frequency $\geq 10, \mathrm{MI} \geq 3$ ). The top 20 collocates in the order of MI score are disgusting, gorgeous, ridiculous, stunning, unacceptable, outrageous, fabulous, absurd, essential, imperative, horrific, delighted, necessary, delicious, correct, crucial, insane, true, amazing, devastating. The overlapping collocates are necessary and right between Middle School and COCA, essential, necessary and wrong between College and COCA and necessary and different between Middle School and College, which means that obvious difference exists in the use of absolutely between Chinese learners and natives. On the one hand, Chinese learners underuse the collocates of absolutely, on the other hand, the difference in the most frequently used collocates explains that the semantic relation between the node and the collocates is different.

The collocates in COCA can be categorized semantically into three groups. The first conveys the meaning of "necessity", including essential, imperative, crucial, necessary; the second expresses "excellence", including gorgeous, fabulous, delighted, delicious, correct, true, amazing; the third expresses "dislike", including disgusting, ridiculous, stunning, unacceptable, outrageous, absurd, horrific, insane, devastating. Although the semantic preferences here are not consistent with Partington's "hyperbole, superlatives" (Partington, 2004, p. 148), we share a point that absolutely displays a distinct semantic preference in collocating with items which have a strong or superlative sense: among its significant collocates. Coincidently, the first group collocates are all neutral collocates, accounting for $20 \%$, the second all positive collocates, accounting for $35 \%$ and the third negative collocates, accounting for $45 \%$. So different from completely whose negative prosody dominates, absolutely conveys a mixture of semantic prosody.

A comparison between Middle School and College shows that the words co-occurring with absolutely have increased significantly, which means that with the advance of English proficiency, Chinese learners acquire more depth knowledge of absolutely. Besides, only one negative collocate "poor" appears in Middle School, but there are 8 negative collocates in College, taking up around $40 \%$ of the total collocates (wrong, harmful, infeasible, depressing hostile, disastrous, irrational, appalling). Obviously, the use of absolutely in College is more native-like than in Middle school.

Totally 
Different, wrong, pretentious and amazing co-occur with totally in Middle School, but only different (Freq=3, MI=4.2) reaches the criterion for collocate. As Table 3 shows, 22 adjectives appear in the co-text in College and 31 adjective collocates in COCA.

Table 3

Adjectives Co-occurring With Totally in College and COCA

\begin{tabular}{|c|c|c|c|c|c|c|c|c|c|c|c|}
\hline \multicolumn{6}{|c|}{ College } & \multicolumn{6}{|c|}{ COCA } \\
\hline Collocate & Freq & MI & Collocate & Freq & MI & Collocate & Freq & MI & Collocate & Freq & MI \\
\hline Different & 18 & 3.7 & Sick & 1 & 4.7 & Unacceptable & 29 & 7.6 & Innocent & 14 & 4.8 \\
\hline Wrong & 4 & 4.3 & Unforgettable & 1 & 4.2 & Unprepared & 12 & 7.6 & Ridiculous & 11 & 4.6 \\
\hline New & 8 & 3.0 & Familiar & 1 & 4 & Irresponsible & 10 & 6.7 & Normal & 34 & 4.4 \\
\hline Free & 2 & 2.7 & Opposite & 1 & 3.6 & Unrelated & 12 & 6.7 & Convinced & 12 & 4.2 \\
\hline Devoid & 1 & 8.5 & Normal & 1 & 3 & Inappropriate & 27 & 6.6 & Random & 10 & 4.1 \\
\hline Unfavorabl & 1 & 8.2 & Fresh & 1 & 3 & Unaware & 16 & 6.4 & Accurate & 11 & 4.1 \\
\hline Astonished & 1 & 7.5 & & & & Unnecessary & 15 & 6.2 & Fine & 44 & 4.1 \\
\hline Confident & 1 & 6.9 & & & & Unfair & 14 & 5.9 & Confident & 11 & 4.0 \\
\hline Identical & 1 & 6.9 & & & & Unexpected & 24 & 5.7 & Wrong & 52 & 3.9 \\
\hline Puzzled & 1 & 6.4 & & & & Different & 352 & 5.3 & Silent & 12 & 3.8 \\
\hline Tragic & 1 & 6.3 & & & & Honest & 30 & 5.3 & Opposite & 12 & 3.7 \\
\hline Rude & 1 & 6.2 & & & & Awesome & 14 & 5.2 & Comfortable & 13 & 3.6 \\
\hline Immoral & 1 & 6.1 & & & & False & 24 & 5.2 & Safe & 21 & 3.4 \\
\hline Ridiculous & 1 & 6.1 & & & & Dependent & 16 & 5 & Independent & 14 & 3.2 \\
\hline Noisy & 1 & 6.0 & & & & Naked & 18 & 5 & Crazy & 12 & 3 \\
\hline Noble & 1 & 5.1 & & & & Blind & 18 & 5 & & & \\
\hline
\end{tabular}

As shown in Table 3, 3 out of 22 adjectives meet the criterion for collocates in College and only different and wrong appear in COCA, which means that there is a big discrepancy in using totally between Chinese learners and natives. Taking all adjectives into consideration, we find that only 5 words, different, normal, opposite, ridiculous and wrong, overlap in College and COCA. We search the other 17 words respectively in COCA, the results show that 8 words have never co-occurred with totally and 7 words co-occur no more than 3 times with totally. It proves that Chinese learners misuse totally and non-nativeness exists in their interlanguage. Another unexpected phenomenon exposing the tiny difference between synonyms is that devoid only co-occurs with totally 5 times and even cannot be regarded as a real collocate, but it strongly collocates with completely.

In COCA, we find that 11 collocates with high collocability, as the bold-faced words show in Table 3, convey the meaning of "absence", which is consistent with Patington (2004)'s findings. As for semantic prosody, 12 collocates are negative, including unacceptable, unprepared, irresponsible, inappropriate, unfair, unexpected, awesome, false, naked, blind, ridiculous, wrong, accounting for around 40\%; 6 italicized collocates are positive, taking up $20 \%$ and 13 collocates are neutral, taking up around $40 \%$. So we conclude that totally convey a mixed semantic prosody with its high frequency collocates and the strongest collocates are the words with "negation" affix.

\section{High frequency verbs collocating with the synonyms.}

Within 4-4 window span, the author retrieved all verbs in different corpora, and then manually excluded those which don't collocate with the synonyms. As shown in Table 4, 40 verbs collocates with completely, 4 with absolutely and 13 with totally. A comparison of the collocates of the three synonyms finds that almost all 
collocates of totally are shared by completely, whereas in all collocates of absolutely, only agree shared by completely and totally. Therefore, it can be concluded that totally is much more similar with completely than absolutely in the colligation of "Verb + synonym / synonym + Verb", and completely has a wider range of collocation with verbs than the other two synonyms.

Table 4

The Verb Collocates of the Synonyms in COCA

\begin{tabular}{|c|c|c|c|c|c|c|c|c|c|c|c|}
\hline \multicolumn{6}{|c|}{ completely } & \multicolumn{3}{|c|}{ absolutely } & \multicolumn{3}{|c|}{ totally } \\
\hline Collocate & Freq & MI & Collocate & Freq & MI & Collocate & Freq & MI & Collocate & Freq & MI \\
\hline Submerged $*$ & 12 & 5.3 & Overlooked & 11 & 3.9 & Devastated & 21 & 4.8 & Freaked & 17 & 7.2 \\
\hline Healed & 13 & 5.2 & Blocked * & 18 & 3.9 & Terrified & 21 & 4.2 & Disagree & 42 & 5.4 \\
\hline Rebuilt & 10 & 4.7 & Ignore & 25 & 3.8 & Thrilled & 17 & 4.1 & Agree & 134 & 5.1 \\
\hline Ignored & 51 & 4.6 & Wasted * & 10 & 3.7 & Agree & 92 & 3.3 & Overwhelmed & 11 & 4.5 \\
\hline Eliminated & 20 & 4.6 & Ruined * & 10 & 3.7 & & & & Ignored & 21 & 4.3 \\
\hline Ignoring & 23 & 4.6 & Blown away & 13 & 3.6 & & & & Destroyed & 21 & 4.3 \\
\hline Destroyed * & 48 & 4.6 & Transformed & 18 & 3.6 & & & & Forgot & 12 & 4.2 \\
\hline Forgot & 29 & 4.5 & Wiped out & 15 & 3.6 & & & & Shocked & 13 & 3.9 \\
\hline Absorbed & 17 & 4.5 & Exhausted & 13 & 3.5 & & & & Eliminate & 10 & 3.8 \\
\hline Overwhelmed & 20 & 4.4 & Abandoned & 24 & 3.4 & & & & Blew & 10 & 3.6 \\
\hline Disappear & 27 & 4.4 & Vanished & 10 & 3.3 & & & & Committed & 19 & 3.3 \\
\hline Agree & 160 & 4.3 & Destroy * & 16 & 3.3 & & & & Forgotten & 11 & 3.2 \\
\hline Disagree & 38 & 4.3 & Trusted & 11 & 3.3 & & & & Focused & 26 & 3.0 \\
\hline Cool & 150 & 4.3 & Transform & 11 & 3.3 & & & & & & \\
\hline Forgotten & 44 & 4.3 & Isolated & 18 & 3.2 & & & & & & \\
\hline Recovered & 25 & 4.2 & Changed & 87 & 3.2 & & & & & & \\
\hline Collapsed * & 23 & 4.1 & Covered & 53 & 3.1 & & & & & & \\
\hline Eliminate & 24 & 4.1 & Understands & 10 & 3.0 & & & & & & \\
\hline Disappeared & 39 & 4.0 & Shut & 42 & 3.0 & & & & & & \\
\hline Melted & 17 & 4.0 & Understood & 34 & 3.0 & & & & & & \\
\hline
\end{tabular}

The collocates of completely can be generally divided into two major types, the first is "absence" collocates labeled by the bold-faced words; the second is "change" collocates represented by italicized words in Table 4 . Totally is similar with completely in semantic preference, but absolutely is different from them. Although it has $17.5 \%$ probability to express negative prosody marked by 7 collocates with ${ }^{*}$, completely conveys mainly a neutral prosody in this colligation because of the majority of neutral collocates. However, absolutely mainly conveys negative prosody when it frequently co-occurs with negative collocates like devastated, terrified and thrilled.

As shown in Table 5, in Chinese learner interlanguage, especially in College, although there are many verbs co-occurring with the synonyms within a 4-4 span, but a few verbs meet the default collocation criterion (frequency $\geq 3, M I \geq 3$ ), Which means that Chinese learners have not established a fixed relation between the synonyms and a set of semantic related words such as "absence" or "change" collocates and they randomly select a word to collocate with the synonym. Taking all co-occurring verbs into consideration, only several verbs overlap between Chinese learners' language and native speaker language and many verbs used by native speakers to collocate with the synonyms don't appear in COCA at all. 
Table 5

Verbs Co-occurring With the Synonyms in DIfferent Corpora

\begin{tabular}{lcccc}
\hline & & College & Middle School \\
\cline { 2 - 5 } Completely & Co-occurring & Verb collocates & Co-occurring Verbs & Verb \\
Totally & 67 & Replace, Agree & 15 & Change \\
Absolutely & 33 & Immersed, Disagree, Agree, Change, & 7 & 0 \\
\hline
\end{tabular}

A comparison of the co-occurring verbs of the three synonyms shows that only two verbs are shared by all the three synonyms and 13 verbs overlap between completely and totally, which is, to a certain extent, consistent with COCA. In many situations, completely and totally are interchangeable in many situations, but absolutely shows a distinct collocates and semantic preference.

\section{Conclusion and Implications}

This study applies corpus-driven approach to investigate the acquisition of completely, absolutely and totally by comparing Chinese learners' interlanguage and native speakers' English based on Co-selection Theory. The results demonstrate Chinese learners' acquisition feature of the group of synonyms.

(1) In comparison with native speakers, not only do the synonyms are less used by Chinese learners but also less adjectives and verbs co-occur with the synonyms and only a few co-occurring words meet the criterion for collocates, which exposes that Chinese learners underuse the synonyms and have not established a fixed semantic relation between the node and its collocates. The reason for this phenomenon may be that Chinese learners use less adverbs to intensify and enrich their writing than natives.

(2) Many co-occurring words used by Chinese learners don't appear in COCA, which shows that Chinese learners misuse the synonyms. Under the traditional and dominating slot-and-filler grammar's influence, Chinese learners pay more attention to the correctness of colligation and use some grammatically correct but non-native even wrong collocations.

(3) The semantic preference and semantic prosody of the synonyms shown in Chinese learners' interlanguage is not consistent with natives'. On the one hand, comparatively low frequency of absolutely and totally in interlanguage corpora means a small number of co-occurring words, which hardly forms fixed semantically relations between collocates, on the other hand, the co-occurring words used by Chinese learners are so diverse that no meaning is shared by a group of collocates. Chinese learners' random selection of collocates naturally results in the unusual collocations, inconsistent semantic preference and semantic prosody. Of course, these random collocations results from Chinese interference, that is, intralinguistic transfer. For example, "absolutely convince" is the translation of "jue dui xiang xin", "totally identical" from "wan quan yi zhi".

(4) With the advance of English proficiency level, more words are selected to co-occur with the synonyms, but some of the newly-used words are not or seldom used by native speakers. Besides, College learners don't show clear and significant improvement in building and distinguishing semantic preference and semantic prosody of the synonyms in contrast to middle school learners. These features show that 
Chinese learners have not fully acquired the use of the group of synonyms, especially the tiny difference among them.

In order to promote the acquisition of synonyms, especially grasp the tiny and subtle difference among synonyms, the focus of acquisition should shift from single word to its syntactic relations, namely, its typical collocation, colligation, semantic preference and semantic prosody. Additionally, textbooks should gradually present the most frequently used collocates and help learners categorize collocates through exercises or tasks. Only in this way can the nativeness of learners' interlanguage be substantially increased.

\section{References}

Conzett, J. (1997). Integrating collocation into a reading and writing course. In J. Coady \& T. Huckin (Eds), Second language vocabulary acquisition. Cambridge: Cambridge University Press.

FANG, Z. C. (2012). Syntactic preference and meaning of collocation. CAFEL, 9, 18-22.

Firth, J. (1957). Papers in linguistics. Oxford: Oxford University Press.

Gardiner, M. \& Dras, M. (2007). Corpus statistics approaches to discriminating among near-synonyms. Proceedings of the 10th Conference of the Pacific Association for Computational Linguistics, 31-39.

Hoey, M. (1991). Patterns of lexis in text. Oxford: Oxford University Press.

HU, H. C. M. (2015). A semantic prosody analysis of three adjective synonymous Pairs in COCA. Journal of Language \& Linguistic Studies, 11, 117-130.

Hunston, S. (2002). Corpora in applied linguistics. Cambridge: Cambridge University Press.

Hunston, S., \& Thompson, G. (Eds.). (2000). Evaluation in text: Authorial stance and the Construction of discourse. Oxford: Oxford University Press.

Kayaoğlu, N. M. (2013). The use of corpus for close synonyms. Journal of Language \& Linguistic Studies, 9, 128-141.

Lewis, M. (1993). The lexical approach: The state of ELT and the way forward. Hove: Language Teaching Publications.

Louw, B. (1993). Irony in the text or insincerity in the writer? The diagnostic potential of semantic Prosodies. In M. Baker, G. Francis, \& E. Tognini-Bonelli (Eds.). Text and technology: In honour of John Sinclair. Amsterdam: John Benjamins.

LU, J. (2010). A corpus-based study on collocational behavior and semantic prosody of near synonyms in Chinese learner English. Modern Foreign Languages, 3, 276-284.

PAN, F. (2010). Lexical acquisition viewed from a contrastive analysis of collocational behavior of near synonyms. Chinese Journal of Applied Linguistics, 5, 52-63.

PANG, Y. \& YANG Y. C. (2012). A corpus-based study of lexical priming of synonymy: Taking begin, start and commence as examples. Foreign Languages and Their Teaching, 6, 21-25.

Partington, A. (1998). Patterns and meanings: Using corpora for English language research and teaching. Amsterdam: John Benjamins Publishing Company.

Partington, A. (2004). Utterly content in each other's company: Semantic prosody and semantic preference. International Journal of Corpus Linguistics, 14, 131-156.

Quirk, et al. (1985). A comprehensive grammar of the English language. London: Longman.

Sinclair, J. (1996). The search for units of meaning. Textus, 9, 75-106.

Sinclair, J. (2003). Reading concordances: An introduction. London: Pearson Longman.

Sinclair, J. (2008). The phrase, the whole phrase and nothing but the phrase. In S. Granger, \& F. Meunier, An interdisciplinary perspective. Amsterdam/Philadelphia: John Benjamins.

Stubbs, M. (1996). Text and corpus analysis. Oxford: Blackwell Publishers Ltd.

Tognini-Bonelli, E. (2001). Corpus linguistics at work. Amsterdam/Philadelphia: John Benjamins Publishing Company.

WEI, N. X. (2006). A corpus-based contrastive study of semantic prosodies in leaner English. Foreign Language Research, 5 , 50-54.

XIAO, R. \& McEnery, A. (2006). Collocation, semantic prosody, and near synonymy: A cross-linguistic perspective. Applied Linguistics, 27, 103-129.

ZHANG, J. D., \& LIU, P. (2005). Corpus-based approaches to the differentiation of English synonyms. Journal of Pla University of Foreign Languages, 6, 49-52. 